\title{
Dual Autocollimators Reading Type High Accuracy Zero Position Variation Detection Method
}

\author{
Haipeng Zhang, Jinsong Wang, Feng Wang, Mai Hu \\ Changchun University of Science and Technology, \\ Jilin, Changchun 130022, China \\ 327161705@qq.com, soldier_1973@163.com
}

\begin{abstract}
In this paper, in view of the influence of shimmer sights detection in mirror installation on the adjusting frame position and shape, Research on high-precision zero detection method can eliminate shimmer sights chucking errors. The method uses a CCD camera to measure the total of zero change, dual autocollimator make shimmer sights chucking generated mounts shape change and repeat chucking difference quantitative detection, and were excluded from the CCD measured values, finally obtained a pure zero change . After verification, the value of not more than $6.48^{\prime \prime}$.
\end{abstract}

Keywords-shimmer sights; double autocollimators; repetitive positioning difference; $C C D$ image measuring

\section{INTRODUCTION}

Zero position variation of the sights is the main factor that influence the firing accuracy of weapon, it is one of the projects that must be checked before the sight experiment, and it is also an important indicator of the stability evaluation of sight.

The Shimmer sights in this paper consist of the adding micromirror before the white sight, so we need to detect the Zero position variation of white light collimator and Shimmer group, The design of the product requires the variation is pure sight of its own, not including the clamping error of the sight and Sighting equipment fixture configuration changes caused by assembling. According to the research, At present the detection of Zero position variation only on the whole Zero position variation detection, can not remove the clamping error of the sight and Sighting equipment fixture configuration changes caused by assembling. Taking into account the development of the sight is the real demand, It is necessary to expand the study.

\section{THE MEASURING PRINCIPLE}

\section{A. Digital Readout Measuring Zero Position Variation Measurement}

We know that to measure the sight Zero position variation must establish an infinity reference target, usually with parallel light tube to simulate. As shown in figure $1.1, R_{g}$ is parallel light pipe reticle, $L_{g}$ is autocollimator objective lens, $R_{0}$ is sight reticle, $L_{0}$ is sight objective lens, $L_{1}$ is sight eyepiece lens, $L_{c}$ is measurement CCD objective lens. $R_{c}$ is measurement $\mathrm{CCD}$ physiognomy. autocollimator reticule center $\mathrm{O}$ In sight reticle image on is A, while the gun's sight reticle center $\mathrm{O}_{0}$ corresponding to the object space point in the collimator reticule $O_{c}$.From the figure geometric relationship show: $\tan \theta=\frac{O O_{c}}{O O_{g}}=\frac{O O_{c}}{f_{g}^{\prime}}$

$\theta=\arctan \frac{O O_{c}}{f_{g}^{\prime}}$

Because of

$$
\frac{O O_{c}}{f_{g}^{\prime}}=\frac{O^{\prime} O_{c}^{\prime}}{f_{c}^{\prime}}
$$

And so

$$
O o_{c}=\frac{f_{g}^{\prime}}{f_{c}^{\prime}} \cdot o^{\prime} o_{c}^{\prime}
$$

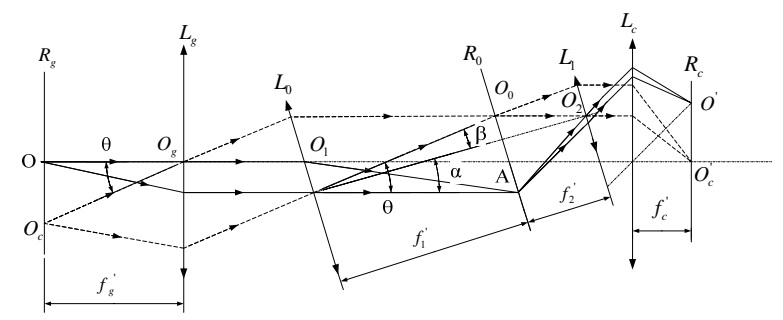

Figure 1.1. Digital readout type zero measurement schematics move

In the formula, $f_{g}^{\prime}$ is collimator lens focal length, $f_{c}^{\prime}$ is Measurement of CCD lens focal length, $\frac{f_{g}^{\prime}}{f_{c}^{\prime}}$ can be detected in advance. $O^{\prime} O_{c}^{\prime}$ Can be measured by the CCD measurements and obtained by image processing. In formula (1) or (2) for the total zero position variation $\theta$.

\section{B. Repetitive Positioning Error Eliminating}

Gun's sights and sight installation connections generally adopt the disassembly convenient pickup or positive definite connection, such as measured by the 
formula (1) results in both pure sight go zero momentum including sight again repeated card error, repeat card error for modern sight products it is more than product request for momentum is zero, so we need to eliminate repetitive positioning error. Method as shown in figure 1.2, $R_{g c}$ for optoelectronic autocollimator CCD image plane; B for half the lens. Optoelectronic autocollimator reticle center; $\mathrm{O}$ by half the lens component in optoelectronic autocollimator, like on the surface of the CCD as for the sight $O^{\prime}$, after repeated loading card mechanical axis and detection system of main axis a (that is, repeat card error), by the geometric relationship is: $\tan 2 \alpha=\frac{o^{\prime} o_{g c}}{O_{g} O_{g c}}=\frac{o^{\prime} o_{g c}}{f_{g}^{\prime}} ; \alpha=\frac{1}{2} \arctan \frac{o^{\prime} o_{g c}}{f_{g}^{\prime}}$

In the formula, $O^{\prime} O_{g c}$ can be read by optoelectronic autocollimator. $\alpha$ can adjust the frame of attitude change in beta in (1) measured $\theta$ shall be excluded.

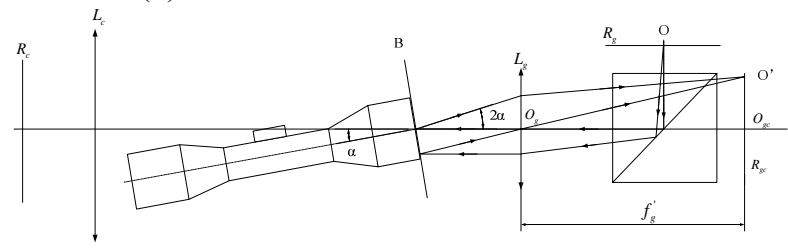

Figure 1.2. Half the semi-permeable eliminate duplicate card error principle diagram
Shimmer sights (white light sights + shimmer group) when detecting micro lens assembly cards to adjust the plane of the shape and position of the impact, which leads to adjust the attitude changes and the introduction of measurement error, the measurement result includes three parts: go sight zero momentum、 sight repeat card error and installation micro lens group after the adjustment of attitude change. So you need to install after micro lens group to measure and adjust the frame of attitude change shall be excluded. The method is shown in figure 1.3, $R_{g 1}$ for optoelectronic autocollimator 2 reticle; $R_{g c 1}$ as the $2 \mathrm{CCD}$ optoelectronic autocollimator face reading; $L_{g}$ for optoelectronic autocollimator 2 lens; F plane mirror; Optoelectronic autocollimator 2 reticle center $O_{1}$ by half the lens component in optoelectronic autocollimator $2 \mathrm{CCD}$ as like to $\mathrm{O}_{1}{ }^{\prime}$, on the surface of the packed card micro lens group after the plane mirror and optoelectronic autocollimator 2 axis vertical plane Angle (adjust shape variation) for $\beta$, known by geometric relations: $\quad \tan 2 \beta=\frac{o_{1}^{\prime} o_{g c 1}}{o_{g 1} O_{g c 1}}=\frac{o_{1}^{\prime} O_{g c 1}}{f_{g 1}^{\prime}}$ $\beta=\frac{1}{2} \arctan \frac{o_{1}^{\prime} o_{g c 1}}{f_{g 1}^{\prime}}$

\section{Installation Micro Lens Group to Eliminate the Error}

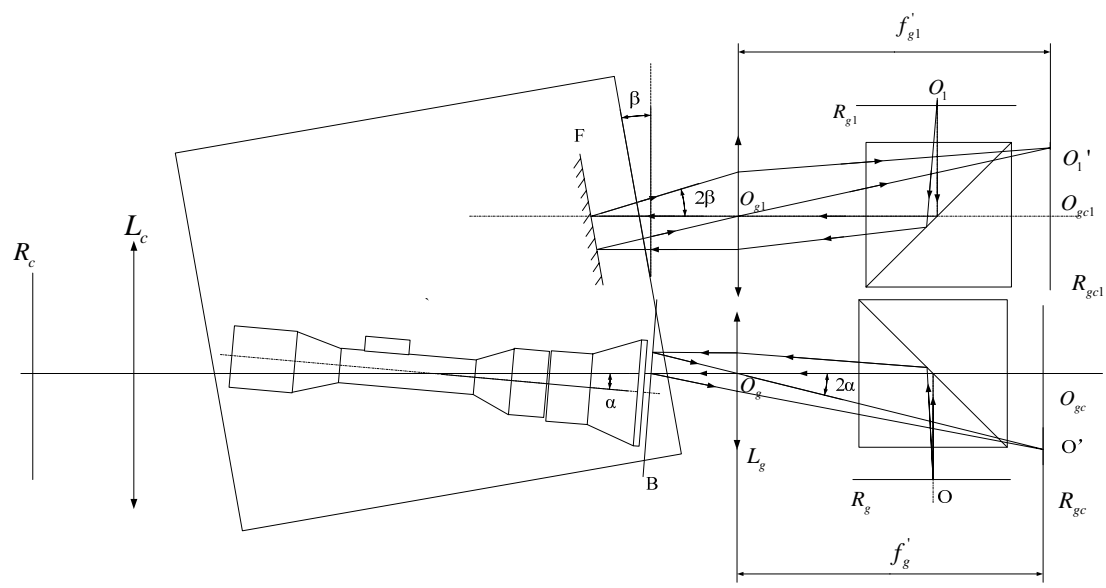

Figure 1.3. card micro lens group to eliminate the error of the principle

Similarly, $\beta$ can adjust the frame of attitude change in beta in (1) measured $\theta$ shall be excluded.

\section{THE IMPLEMENTATION PROCESS}

From the above analysis, to move a pure measurement of the zero position variation, need two sets of photoelectric autocollimator, A measurement was repeated chucking error $\alpha$, And cater to simulate infinity collimator target. Another measure due to the loading rack shimmer mirror group lead to adjustments in the amount of change in attitude $\beta$. So the zero position variation the entire amount is: $\theta-\alpha-\beta$; White light sight collimator zero position variation: $\theta_{1}-\alpha$ Shimmer mirror group zero position variation: $\theta_{2}=\theta-\left(\theta_{1}-\alpha\right)-\beta$

Figure 2.1 experimental device for three-dimensional map. White sight mounted on the adjusting rack, Adjust the rack to make the white light sight cross points, lines and optoelectronic autocollimator 1 cross line close to overlap. This regulation of plane mirror two-dimension adjustment frame, so that the photoelectric autocollimator 
2 reticle after reflection from a plane mirror image and the original photoelectric autocollimator 2 reticle coincidence,

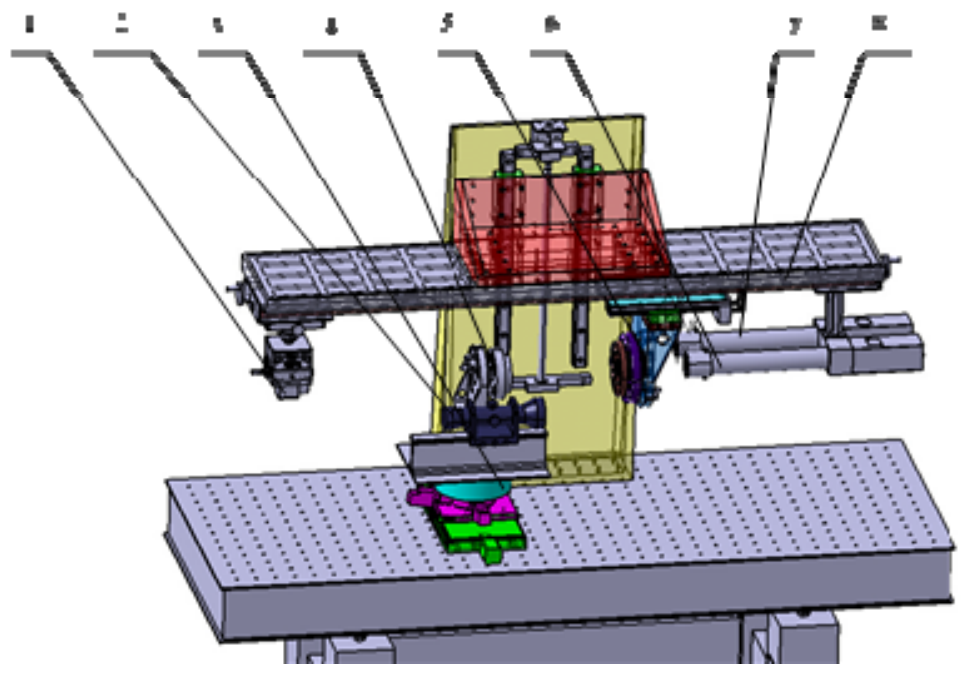

Figure 2.1. system of three dimensional map

1 measurement of CCD,2. The sight,3 adjusting rack,4. The plane mirror component,5. Half the lens component,6. Optoelectronic autocollimator 1,7.Optoelectronic autocollimator 2,8. Lifting platform

Assemble the shimmer mirror group on the front end of the white light sight. Adjust the adjustment frame so that the image after reflection from a plane mirror of the photoelectric autocollimator 2 reticle will re coincidence with the original photoelectric autocollimator 2 reticle. This reflective reticle position reset through the flat mirror, can remove influence on the adjusting frame during the process of assembling shimmer mirror group. Use the two-dimensional with force feedback push the half mirror fit in the front end surface of shimmer lens group object frame. Write down the reflection reticle that photoelectric autocollimator 1 through half mirror and the offset $\theta_{\mathrm{X} 1}$ 、 $\theta_{\mathrm{Y} 1}$ of photoelectric autocollimator 1 original cross lines, and by measuring the CCD and related software program measuring the offset that white sight reticle with respect to the photoelectric autocollimator 1 reticle $\theta_{\mathrm{x} 1} 、 \theta_{\mathrm{y} 1}$ 。

Re-install the white sighting telescope and shimmer mirror group that after test. Adjust the adjusting mount, making photoelectron autocollimator 2 reflected cross engraved lines by the reflector coincide with Original photoelectron autocollimator 2 cross engraved lines. Then there are two cases in phase CCD surface:

1) Photoelectric autocollimator's cross engraved lines and white sighting telescope cross engraved lines are in the field of view measuring CCD. Using 2D adjusting mount with force feedback to make half mirror push fit in front end surface of the shimmer mirror group object frame. Note the time of photoelectron collimator 1 reflected cross engraved lines by the reflector offset to the original photoelectron autocollimator 1 cross engraved lines is $\theta_{\mathrm{X} 2} 、 \theta_{\mathrm{Y} 2}$, and by measuring the CCD and associated software to measure the offset photoelectron autocollimator 1 reflected cross engraved lines by the reflector offset to the original photoelectron autocollimator 1 cross engraved lines is $\theta_{\mathrm{x} 2} 、 \theta_{\mathrm{y} 2}$ 。

2) Cross engraved lines of sight are not in the field of view of measuring CCD. Now sight's repeat chucking error is greater than the measurement range of the measurement CCD. Adjust mounts to make sight's cross engraved lines back to the field of view of measuring CCD, excluding partial duplicate chucking error by adjusting the adjusting mount. And repeat 1) process.

Make the before test and after test subtraction by $\mathrm{CCD}$ detector to measure sights: $\Delta \theta_{X}=\theta_{X 2}-\theta_{X 1}$ and $\Delta \theta_{Y}=\theta_{Y 2}-\theta_{Y 1}$.

This part of the result of the error includes chucking error, and includes sight pure zero change. The repeating errors of chucking can get by he offset photoelectron autocollimator 1 reflected cross engraved lines by the reflector offset to the original photoelectron autocollimator 1 cross engraved lines: $\Delta \theta_{x}=\theta_{x 2}-\theta_{x 1}$ and $\Delta \theta_{y}=\theta_{y 2}-\theta_{y 1}$.

\section{THE EXPERIMENTAL AND DATA ANALYSIS}

\section{A. Excluding Installation Error}

Figure 3.1 shows the experimental setup, Through experiments, Verified using this method to remove the repeating errors of chucking is feasible. Can measure CCD photoelectric autocollimator and Measurement CCD camera's readings, by subtraction, As shown in Table I. 


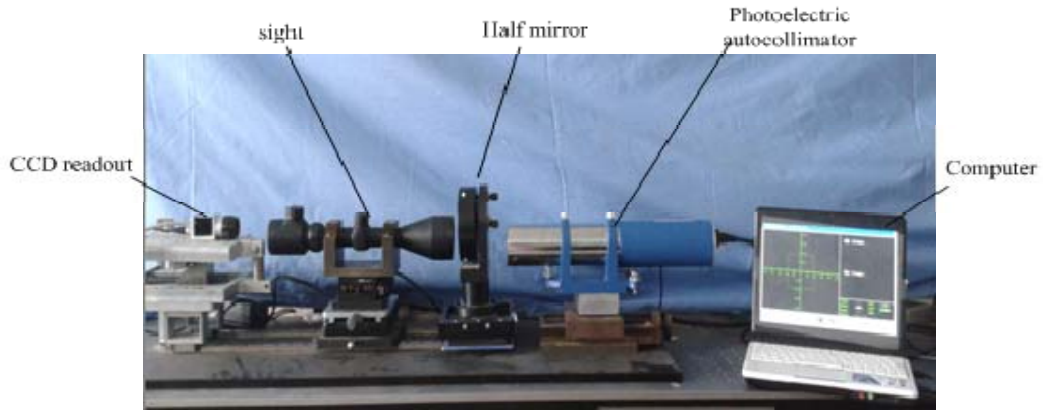

Figure 3.1. experimental setup

TABLE I. READING THE RESULTS

\begin{tabular}{|c|c|c|c|c|c|c|}
\hline & \multicolumn{2}{|c|}{ Reading CCD camera readout } & \multicolumn{2}{|c|}{ CCD photoelectric autocollimator readings } & \multicolumn{2}{|c|}{ The difference between the two } \\
\hline No. & $\Delta \theta_{X}$ & $\Delta \theta_{Y}$ & $\Delta \theta_{x}$ & $\Delta \theta_{y}$ & $\Delta \theta_{X}-\Delta \theta_{X}$ & $\Delta \theta_{Y}-\Delta \theta_{y}$ \\
\hline 1 & $1^{\circ} 13^{\prime} 42.6^{\prime \prime}$ & $-1^{\circ} 11^{\prime} 29.46^{\prime \prime}$ & $1^{\circ} 13^{\prime} 21.0^{\prime \prime}$ & $-1^{\circ} 12^{\prime} 35.3^{\prime \prime}$ & $21.60^{\prime \prime}$ & $65.84^{\prime \prime}$ \\
\hline 2 & $-1^{\circ} 21^{\prime} 30^{\prime \prime}$ & $-1^{\circ} 48^{\prime} 20.43^{\prime \prime}$ & $-1^{\circ} 21^{\prime} 54^{\prime \prime}$ & $-1^{\circ} 49^{\prime} 23.7^{\prime \prime}$ & $24.00^{\prime \prime}$ & 63.27" \\
\hline 3 & $-2^{\circ} 25^{\prime} 34.2^{\prime \prime}$ & $-2^{\circ} 22^{\prime} 9.86^{\prime \prime}$ & $-2^{\circ} 25^{\prime} 54.6^{\prime \prime}$ & $-2^{\circ} 23^{\prime} 9.2^{\prime \prime}$ & $20.40^{\prime \prime}$ & $59.34 "$ \\
\hline 4 & $-2^{\circ} 28^{\prime} 13.6^{\prime \prime}$ & $-1^{\circ} 4^{\prime} 56.93^{\prime \prime}$ & $-2^{\circ} 28^{\prime} 36.4^{\prime \prime}$ & $-1^{\circ} 6^{\prime} 4.5^{\prime \prime}$ & $22.80^{\prime \prime}$ & $67.57^{\prime \prime}$ \\
\hline 5 & $-2^{\circ} 3^{\prime} 33.12^{\prime \prime}$ & $-1^{\circ} 4^{\prime} 56.93^{\prime \prime}$ & $-2^{\circ} 3^{\prime} 59.4^{\prime \prime}$ & $-2^{\circ} 4^{\prime} 39.7^{\prime \prime}$ & $26.28^{\prime \prime}$ & $63.27^{\prime \prime}$ \\
\hline Mean & $-1^{\circ} 25^{\prime} 1.66^{\prime \prime}$ & $-1^{\circ} 30^{\prime} 22.72^{\prime \prime}$ & $-1^{\circ} 25^{\prime} 24.68^{\prime \prime}$ & $-1^{\circ} 55^{\prime} 0.48^{\prime \prime}$ & $23.016^{\prime \prime}$ & $63.858^{\prime \prime}$ \\
\hline RMS $\sigma$ & & & & & $2.26^{\prime \prime}$ & $3.11^{\prime \prime}$ \\
\hline
\end{tabular}

Experimental data obtained by the $s$ value is not greater than $6.48^{\prime \prime}(0.03 \mathrm{mil})$, The test data is reliable, and fully meet the military requirements.

B. Shimmer Group Frame Installation Lead to the Shape Changes and Position of Adjustment Measurement
Experiment, after the installation shimmer mirror group adjusting mount position change beyond the high accuracy requirement. Table II is after the installation shimmer Mirror Group adjusting mount position change measured data.

TABLE II. PHOTOELECTRIC AUTOCOLLIMATOR 2READINGS RESULTS

\begin{tabular}{|c|c|c|c|}
\hline No. & First position & Installation shimmer Mirror Group & After installing shimmer mirror group deformed $\Delta$ \\
\hline 1 & $\mathrm{X}: 399.6^{\prime \prime} ; \quad \mathrm{Y}: 477.4^{\prime \prime}$ & $\begin{array}{l}\mathrm{X}: 643.1^{\prime \prime} \\
\mathrm{Y}: 605.7^{\prime \prime}\end{array}$ & $\mathrm{X}: 243.5^{\prime \prime} ; \quad \mathrm{Y}: 128.3^{\prime \prime}$ \\
\hline 2 & $\mathrm{X}: 406.1^{\prime \prime} ; \quad \mathrm{Y}: 484.3^{\prime \prime}$ & $\begin{array}{l}\text { X: 649.3" } \\
\text { Y: } 610.9^{\prime \prime}\end{array}$ & $\mathrm{X}: 243.2^{\prime \prime} ; \mathrm{Y}: 126.6^{\prime \prime}$ \\
\hline 3 & $\mathrm{X}: 408.1^{\prime \prime} ; \mathrm{Y}: 484.0^{\prime \prime}$ & $\begin{array}{c}\text { X: 649.9" } \\
\text { Y: 611.1" }\end{array}$ & $\mathrm{X}: 241.8^{\prime \prime} ; \mathrm{Y}: 127.1^{\prime \prime}$ \\
\hline 4 & $\mathrm{X}: 407.7^{\prime \prime} ; \mathrm{Y}: 486.2^{\prime \prime}$ & $\begin{array}{l}\mathrm{X}: 650.0^{\prime \prime} \\
\mathrm{Y}: 614.6^{\prime \prime}\end{array}$ & $\mathrm{X}: 242.3^{\prime \prime} ; \mathrm{Y}: 126.6^{\prime \prime}$ \\
\hline 5 & $\mathrm{X}: 405.3^{\prime \prime} ; \mathrm{Y}: 484.9^{\prime \prime}$ & $\begin{array}{l}\text { X: 653.8" } \\
Y: 618.6^{\prime \prime}\end{array}$ & $\mathrm{X}: 248.5^{\prime \prime} ; \mathrm{Y}: 133.7^{\prime \prime}$ \\
\hline
\end{tabular}

As can be seen from the experimental data after the installation shimmer mirror group adjusting mount position change. This part of the error has been exceeded high accuracy sight pure zero change's requirement, so it must be removed.

\section{Error Analysis}

The main factors of error has the following points
1) Calibration error; Due to the human eye alignment error in the calibration process, that is not with the CCD image plane mirror surface coincide, and is not perpendicular to the axis.

2) The CCD and optoelectronic autocollimator reading error due to there is the spacing and the size of CCD photosensitive pixels for the CCD readout process, and adopts the error introduced by binarization processing 
3) Half the lens repeat alignment error; Due to half the lens alignment needs to contact with the front facet of sight, After bonding is different from the first of errors arising.

\section{CONCLUSION}

Using dual autocollimator to realize the shimmer sight line alteration detection with high accuracy. It can eliminate the influence of adjusting frame configuration changes which is caused by repeated chucking error and shimmer group installation. We construct a prototype to achieve the implementation equations of testing and function, we have detected adjusting frame configuration changes which is caused by repeated chucking error and shimmer mirror group installation. The results showed that the double autocollimator method can effectively eliminate the impact of the above errors, and achieve second-level precision testing.
VI. CORRESPONDING AUTHOR

Name: Zhang Haipeng

Email:327161705@qq.com; Mobile phone: 18943977266

\section{REFERENCES}

[1] Wang Jinsong, An Zhiyong, Li Hailan. Research on the measuring method to the IR aiming sight's line alteration of reflection type collimator[J].Acta ArmamentarII,2010, 31(11):1422-1425.

[2] Lv Bo, Han GuoHua, Zhang Aili, Jiang XianDong. Study on measurement of sight-line alteration of high precision sighting telescopes[J]. Laser Technology. 2013(3).

[3] Zhang MingPing, Zhang JingXian, Li YuDan. Night Vision System[M]. Beijing, Beijing Institute of Technology Press. 1993.

[4] SHEN Y Ch, HU B,PANG D,et al. GJB5815-2006. The approval test methods of optical sight for small arms[S].Beijing; General Armament Department Military Standardization Research Center,2006;7-11(in Chinese). 